\title{
Potential Room Temperature Superconductivity in Clathrate Lanthanide/Actinides Octadechydrides at Extreme Pressures
}

\section{Xin Zhong}

Jilin University

\section{Ying Sun}

Jilin University https://orcid.org/0000-0003-1401-107X

\section{Toshiaki litaka}

RIKEN https://orcid.org/0000-0002-7191-7516

\section{Meiling Xu}

Jiangsu Normal University https://orcid.org/0000-0001-6592-8975

Hanyu Liu ( $\sim$ hanyuliu@jlu.edu.cn )

Jilin University

\section{Changfeng Chen}

University of Nevada

\section{Yanming Ma}

Jilin University https://orcid.org/0000-0003-3711-0011

\section{Article}

\section{Keywords:}

Posted Date: December 9th, 2021

DOI: https://doi.org/10.21203/rs.3.rs-1148583/v1

License: (c) (i) This work is licensed under a Creative Commons Attribution 4.0 International License. Read Full License 


\section{Abstract}

Atomic metallic hydrogen $(\mathrm{AMH})$ hosting high-temperature superconductivity has long been considered a holy grail in condensed matter physics and attracted great interest, but attempts to produce AMH remain in intense exploration and debate. Meanwhile, hydrogen-rich compounds known as superhydrides offer a promising route toward creating AMH-like state and property, as showcased by the recent prediction and ensuing synthesis of $\mathrm{LaH}_{10}$ that hosts extraordinary superconducting critical temperatures $\left(T_{c}\right)$ of 250$260 \mathrm{~K}$ at 170-190 GPa. Here we show via advanced crystal structure search a series of hydrogensuperrich clathrate compounds $\mathrm{MH}_{18}$ ( $\mathrm{M}$ : rare-earth/actinide metals) comprising $\mathrm{H}_{36}$-cage networks, which are predicted to host $T_{c}$ up to $329 \mathrm{~K}$ at $350 \mathrm{GPa}$. An in-depth examination of these extreme superhydrides offers key insights for elucidating and further exploring ultimate phonon-mediated superconductivity in a broad class of AMH-like materials.

\section{Introduction}

First predicted 86 years ago by Wigner and Huntington, ${ }^{1}$ metallic hydrogen has been attracting great interest, especially after the conjecture of its ability to host high-temperature superconductivity. ${ }^{2-5}$ The quest for ultimate atomic metallic hydrogen (AMH), however, has proven extremely challenging due to stringent synthesis requirements of ultrahigh pressures and supersensitive characterizations that push experimental limits. ${ }^{6-8}$ An early study of $\mathrm{Th}_{4} \mathrm{H}_{15}$ found this metal hydride superconducting with a critical temperature $T_{C}$ of $8 \mathrm{~K}$ at ambient pressure; ${ }^{9}$ a proposal was also made to use chemical pre-compression to stabilize hydrogen-containing compounds that may host metallic and superconducting hydrogen states. ${ }^{10}$ This line of work has been most actively pursued after Ashcroft's suggestion ${ }^{11}$ that hydrogen dominant metallic alloys may serve as surrogate materials for $\mathrm{AMH}$ in probing ultimate phonon-mediated superconductivity. Recent years have seen concerted efforts in exploring compressed metal hydrides. ${ }^{12-}$ ${ }^{14}$ Hydrogen sulfides with $T_{C}$ of $203 \mathrm{~K}$ at $150 \mathrm{GPa}$ was discovered ${ }^{15}$ following theoretical predictions of high- $T_{\mathrm{C}}$ superconductivity in $\mathrm{H}_{2} \mathrm{~S}^{16}$ and $\mathrm{H}_{3} \mathrm{~S} .{ }^{17}$ On the other route, a distinct class of clathrate superhydrides has been predicted to possess higher $T_{\mathrm{c}}$ values ${ }^{18-20}$ with $\mathrm{CaH}_{6}$ being the first of such examples ever predicted, ${ }^{21}$ culminating with the experimental realization of $\mathrm{LaH}_{10}$ that exhibit high $T_{c}$ of 250-260 K at pressures of 170-190 GPa, ${ }^{22,23} \mathrm{YH}_{9}$ with a $T_{\mathrm{c}}$ of $243 \mathrm{~K}$ at $201 \mathrm{GPa},{ }^{24} \mathrm{YH}_{6}$ with a $T_{\mathrm{c}}$ of $220 \mathrm{~K}$ at $166 \mathrm{GPa}$ or $237 \mathrm{GPa}^{24,25}$ and $\mathrm{CaH}_{6}$ with a $T_{\mathrm{c}}$ of $215 \mathrm{~K}$ at $170 \mathrm{GPa} .{ }^{26,27}$ Other notable cases for clathrate structured high- $T_{c}$ superhydrides include $\mathrm{CaH}_{6},{ }^{21} \mathrm{YH}_{10},{ }^{19,20} \mathrm{Li}_{2} \mathrm{MgH}_{16},{ }^{28}$ and $\mathrm{CaYH}_{12},{ }^{29,30}$ from theory and $\mathrm{ThH}_{9}$ and $\mathrm{ThH}_{10}{ }^{31}$ from experiment. An overriding idea in this area of research is to raise the hydrogen content so that the superhydrides may more closely resemble $\mathrm{AMH}$ in bonding environments and physical properties. A major task is to find superhydrides with proper metal elements that are capable of holding higher hydrogen contents in the nonmolecular form (i.e., hydrogen atomization) that can potentially help generate higher $T_{\mathrm{C}}$ exceeding that of $\mathrm{LaH}_{10} \cdot{ }^{12,21,28,32,33}$ Recent studies ${ }^{14,19,20,31}$ show that rare-earth (RE) and actinide (An) elements are capable of holding a large amount of hydrogen by forming 
clathrate compounds at high pressures, and the resulting hydrides host high- $T_{\mathrm{C}}$ values. These results raise the prospects of finding RE/An hydrides containing even higher hydrogen contents, and such superhydrides may possess further increased $T_{c}$ approaching or even exceeding room temperature. Based on these considerations, we have chosen a series of electron-rich rare-earth and actinide elements as hosts to provide electrons to dissociate molecular hydrogen pairs, thereby creating an AMH-like environment conducive to harboring higher $T_{c}$ superconductivity.

Here, we report on the finding of a series of hydrogen-superrich $\mathrm{MH}_{18}$ compounds, where $\mathrm{M}$ stands for $\mathrm{RE}$ or An elements, identified using our developed structural search algorithm. ${ }^{34,35}$ These extreme superhydrides comprise conspicuous $\mathrm{H}_{36}$ clathrate cages providing an excellent platform for probing the effect of rising hydrogen content on superconducting properties in superhydrides. First-principles calculations reveal unexpectedly diverse $T_{\mathrm{C}}$ values among the $\mathrm{MH}_{18}$ compounds that share the same stoichiometry and bonding structure, suggesting intricate underlying mechanisms. Of the identified superhydrides, $\mathrm{CeH}_{18}$ is predicted to exhibit remarkable above-room-temperature high $T_{\mathrm{c}}$ of $329 \mathrm{~K}$ at 350 $\mathrm{GPa}$, while other compounds possess widely variable $T_{c}$ from $50 \mathrm{~K}$ to $321 \mathrm{~K}$. These intriguing results provide a wealth of information on rich material behaviors and key physics insights that allow an indepth study of factors with major influence on approaching ultimate phonon-mediate superconductivity. This work opens a promising path for further exploration of binary and higher order superhydrides that can meet or even exceed $\mathrm{AMH}$ in hosting superconducting states with higher $T_{C}$ values.

\section{Results}

Phase diagram. We have performed structure searches in binary hydrides $\mathrm{MH}_{\mathrm{m}}(m=2-24)$ over a wide range of hydrogen contents to predict stable structures at high pressures. This search process has led to the discovery of a series of hydrogen-superrich compounds. Particularly noteworthy among these are new stoichiometric $\mathrm{Ce}$ and Th superhydrides $\mathrm{Ce} / \mathrm{ThH}_{18}$ that stabilize at experimentally accessible but technically very challenging pressure range around $400 \mathrm{GPa}$ without considering the zero-point energy (ZPE), as shown in Figure 1(a). Further calculations of the formation enthalpy with the inclusion of ZPE show that $\mathrm{CeH}_{18}$ and $\mathrm{ThH}_{18}$ become stable at notably reduced pressures of 315 and $281 \mathrm{GPa}$ (Figure $1(b)$ ), respectively, making the experimental synthesis and characterization more feasible. A systematic comparison of the stability pressure ranges calculated without and with the inclusion of ZPE is given in Figure S1. We examined a broad range of RE and An superhydrides up to $700 \mathrm{GPa}$, and the results reveal that this $\mathrm{MH}_{18}$ stoichiometry is ubiquitous among diverse $\mathrm{RE}$ and $A n$ superhydrides for $\mathrm{RE} / \mathrm{An}=\mathrm{Y}, \mathrm{La}, \mathrm{Ce}$, Ac, and Th, which are stable over variable extended ranges of pressures as shown in Figure 1(b). Details of the computational methods for the structure search and property calculations are provided in the Supplemental Material. ${ }^{36}$

Crystal structures. The predicted extreme superhydrides $\mathrm{MH}_{18}$ contain conspicuous $\mathrm{H}_{36}$ clathrate units in its crystal structures. Figure 2(a) shows the crystal structure that comprises a peculiar three-dimensional hydrogen clathrate structure of space group $F d d d$, where each $\mathrm{M}$ atom is located at the center of a 
clathrate $\mathrm{H}_{36}$ cage that, as shown in Figure 2(c), consists of a $6 \mathrm{H}_{6}$ ribbon-ring structure with two wrinkled $\mathrm{H}_{6}$ hexagons above and below with bridge bonds connecting the $\mathrm{H}_{6}$ hexagons to the $6 \mathrm{H}_{6}$ ribbon-ring structure. At higher pressures, which vary for different $\mathrm{M}$ atoms, this $\mathrm{H}_{36}$ clathrate units rearrange and stabilize in another structure of space group Fmmm shown in Figure 2(b). Below we focus our analysis mainly on $\mathrm{CeH}_{18}$ to showcase its prominent properties while also discussing key data and trends involving other $\mathrm{MH}_{18}$ compounds.

\section{Discussion}

Band/Dos character and Superconductivity of $\mathrm{CeH}_{18}$. To assess superconducting properties of $\mathrm{CeH}_{18}$, we first evaluate its electronic band structure, taking $400 \mathrm{GPa}$ as a representative case study. Calculated results clearly indicate the metallic nature of $\mathrm{CeH}_{18}$ with several band crossing the Fermi level, as shown in the left panel of Figure 3(a). Results in the right panel of Figure 3(a) show that the hydrogen atoms make a substantial contribution to the electronic density of states (DOS) near the Fermi level, which is almost identical to the DOS contributed by the electrons from Ce. It is seen that the DOS is essentially flat around the Fermi energy, which is notably different from the DOS of $\mathrm{LaH}_{10}$ that hosts van Hove singularity around the Fermi energy ${ }^{19}$. To examine the changes of the band-filling states, we have calculated the band structures at different pressures, and the results (see Figure S2) show that the holebands at the $\mathrm{X}$-point, along the $\mathrm{Z}-\mathrm{X}$ path, and at the $\mathrm{Y}$-point move downward with rising pressure, indicating a systematic pressure driven electron transfer, which provides an explanation for the decreasing trend of $T_{\mathrm{C}}$ with increasing pressure. We next examine the phonon and electron-phonon coupling in $\mathrm{CeH}_{18}$. Calculated phonon dispersion results are shown in the left panel of Figure 3(b). No imaginary phonon modes are present in the entire Brillouin zone, indicating the dynamic stability of this crystal structure. We then computed the Eliashberg spectral function $a^{2} F(\omega)$, from which the electronphonon coupling parameter can be obtained via a simple integration in the frequency domain. ${ }^{37-40}$ The resulting integrated electron-phonon coupling parameter $\lambda=2.3$ is quite large and comparable to the value of $\lambda=2.2$ for $\mathrm{H}_{3} \mathrm{~S} .{ }^{17}$ It is noted that such strong electron-phonon couplings make various approximate weak-coupling $T_{\mathrm{C}}$ formulas generally unreliable, and an accurate description necessitates direct numerical solutions to the Eliashberg equations. ${ }^{37-40}$ We have employed this approach to calculate superconducting energy gap and transition temperature using the typical Coulomb pseudopotential $\mu^{\star}=$ 0.10; we also checked results with $\mu^{\star}=0.13$ to estimate a reasonable range of $T_{\mathrm{c}}$ values. The resulting $T_{\mathrm{c}}$ of $309-329 \mathrm{~K}$ (for $\mu^{\star}=0.10$ and 0.13 ) at $350 \mathrm{GPa}$, where $\mathrm{CeH}_{18}$ is stable, is well above the room temperature and represents the highest hitherto reported $T_{\mathrm{c}}$ among binary superhydrides.

Superconductivity of $\mathrm{MH}_{18}$. To explore systematic trends and elucidate the underlying mechanisms for superconductivity in the predicted extreme superhydrides, we have calculated key electronic, phonon and electron-phonon coupling parameters and the resulting $T_{\mathrm{c}}$ values from solving the Eliashberg equations of selected $\mathrm{MH}_{18}$ compounds. We also have calculated, for the purpose of comparison, under the same 
parameters employing the same computational approach for the $14_{1} / a m d$ phase of solid hydrogen that is known to stabilize in the pressure range of interest here. ${ }^{41}$ Moreover, we have systematically examined energetic and phonon dispersion aspects of the $\mathrm{MH}_{18}$ compounds beyond their stability fields indicate in Figure1(b), and we have identified several metastable phases that extend the structural viability to lower pressures with enhanced superconducting transition temperatures. All the key parameters and properties for the identified stable and metastable phases of $\mathrm{MH}_{18}$ and hydrogen in $14_{1} / a m d$ phase are summarized in Table I with the $T_{c}$ data plotted in Figure 4.

Calculated results show that the electron-phonon coupling parameter $\lambda$ and the resulting $T_{\mathrm{c}}$ vary considerably among the $\mathrm{MH}_{18}$ superhydrides at the same pressure points (see Table I and Figure 4). This phenomenon reflects the broadly variable lattice dynamics and electronic states in different $\mathrm{MH}_{18}$ compounds, despite that they all share the same stoichiometry and clathrate structures. These contrasting properties indicate substantial differences in their bonding strengths and charge distributions, which are manifested in the large disparities of their characteristic vibrational frequencies and electronic density of states at the Fermi level as listed in Table I.

It is noted that the $\mathrm{MH}_{18}$ compounds host $T_{\mathrm{c}}$ values that distribute over a large range, from $50 \mathrm{~K}$ for $F d d d-$ $\mathrm{AcH}_{18}$ at $700 \mathrm{GPa}$ up to $329 \mathrm{~K}$ for $F d d d-\mathrm{CeH}_{18}$ at $350 \mathrm{GPa}\left(T_{\mathrm{c}}\right.$ quoted for discussion are all taken at $\mu^{\star}=$ 0.10). These results offer important clues for understanding the trends in $T_{\mathrm{C}}$ values that approach the result of the $14_{1} /$ amd phase of solid hydrogen at high pressures. Among the extreme superhydrides, $\mathrm{CeH}_{18}$ exhibits the highest $T_{\mathrm{C}}$ in the entire pressure range, and this compound hosts $\mathrm{H}-\mathrm{H}$ distances in the range of $0.85-1.17 \AA$ that is close to that of $\mathrm{AMH}(1.0 \AA)$ at $500 \mathrm{GPa}$. These results show that these newly identified extreme $\mathrm{MH}_{18}$ superhydrides represent a series of compounds that approach $\mathrm{AMH}$ in superconducting properties, confirming Ashcroft's original conjecture. ${ }^{11}$ We have calculated the projected phonon density of states (PHDOS) from $\mathrm{Ce}$ and $\mathrm{H}$ atoms. The results given in the middle panel of Figure 3 (b) show that the main contributions to the EPC come from the mid- and high-frequency hydrogen vibrations in the range of $500-2500 \mathrm{~cm}^{-1}$. It is also seen from the results in Figure 4 that most $\mathrm{MH}_{18}$ compounds exhibit monotonically decreasing $T_{c}$ with increasing pressure with the exception of Fddd$\mathrm{ThH}_{18}$ that hosts a slightly upward trend in its $T_{\mathrm{c}}$ versus rising pressure. This pressure induced reduction of $T_{c}$ is reminiscent of the behavior of solid hydrogen at higher pressures $(700-1,000 \mathrm{GPa}){ }_{1}^{5}$ which is consistent with the idea that chemical pre-compression in hydrogen compounds would shift material behaviors toward lower pressures. These insights are helpful for rational exploration and elucidation of optimal superconducting superhydrides.

Taking potential effects into consideration. Additional effects, such as spin-orbit coupling (SOC), magnetism and electron correlation, may affect the estimated $T_{\mathrm{C}}$ of the predicted $\mathrm{MH}_{18}$ compounds. We have taken $\mathrm{CeH}_{18}$ and $\mathrm{ThH}_{18}$ as case studies to assess the effect of SOC. Our calculations reveal that the $T_{\mathrm{C}}$ values are insensitive to SOC (see Table I), which is consistent with the nearly identical band structures with and without the SOC (Figure 3a). We have examined the energetics of the magnetic structures of the 
predicted $\mathrm{MH}_{18}$ compounds by considering six possible magnetic configurations (one ferromagnetic and five antiferromagnetic configurations). The results show that the nonmagnetic state is the most stable for all the predicted $\mathrm{MH}_{18}$ com pounds. We have not considered electron correlation effects on the EPC due to a lack of available computational tools for this purpose. We note, however, that previous studies $^{17,19-21}$ have shown that the experimental results on several superhydrides (e.g., La, Y, Th, and Ca hydrides $^{15,22-25,29,30,42}$ ) are well described by the theoretical results obtained within the current EPC computation scheme without considering the electron correlation effects. On this basis, it is expected that the results in the present work offer a reasonably accurate description of the superconductivity in the newly predicted $\mathrm{MH}_{18}$ compounds.

In summary, we have identified a series of $\mathrm{MH}_{18}$ compounds containing the highest atomic hydrogen content among metal hydrides reported to date. These extreme superhydrides comprise unique $\mathrm{H}_{36}$ clathrate units stabilized at high pressures starting around 300-400 GPa. Favorable conditions of electronic density of states near the Fermi level and lattice vibrational modes that set large phonon energy scale and strong electron-phonon coupling in $\mathrm{CeH}_{18}$ generate high $T_{\mathrm{c}}$ of $329 \mathrm{~K}$ at $350 \mathrm{GPa}$. Meanwhile, other $\mathrm{MH}_{18}$ compounds exhibit a wide range of $T_{\mathrm{c}}$ with variable electronic, phonon and electron-phonon coupling combinations. These results offer insights into intricate mechanisms for superconductivity in superhydrides, establishing a promising platform for further exploration and optimization of diverse superhydrides that approach and may even exceed high-temperature superconductivity predicted for atomic metallic hydrogen.

\section{Methods}

Structural predictions. Our structure search is based on PSO algorithm ${ }^{43,44}$ using the CALYPSO methodology. ${ }^{34,35,45}$ We performed structure searches at $400 \mathrm{GPa}$ with 1- 4 formula units (f. u.) per cell of $\mathrm{MH}_{\mathrm{x}}(x=2-24)$. Most searches converge in 50 generations with about 2,500 structures generated.

Ab initio calculations. Structural optimization and computations of enthalpy, phonon, electronic structures, and spin-orbital coupling (SOC) were all performed in the framework of density-functional theory (DFT) as implemented in the VASP code. ${ }^{46}$ The Perdew-Burke-Ernzerhof ${ }^{47}$ generalized gradient approximation ${ }^{48}$ was employed, and a kinetic cutoff energy of $500 \mathrm{eV}$ was adopted to ensure that the calculated enthalpy converges to better than $1 \mathrm{meV} /$ atom. The ZPE of predicted compounds were obtained from lattice dynamic calculations as implemented the PHONOPY code. ${ }^{49}$

EPC calculations. The electron-phonon coupling (EPC) calculations were carried out using the QUANTUM ESPRESSO code. ${ }^{50}$ Ultrasoft pseudopotentials for RE/An and $\mathrm{H}$ elements were used with a kinetic energy cutoff of 80 Ry. To reliably calculate the electron-phonon coupling in metallic systems, we have employed $k$-meshes of $2 \pi \times 0.045 \AA^{-1}$ for the electronic Brillouin zone integration and $q$-meshes of $2 \pi \times 0.09 \AA^{-1}$ for all the phonon calculations of $\mathrm{MH}_{18}$ compounds. We have employed the Migdal-Eliashberg theory ${ }^{51}$ to calculate superconducting energy gap and transition temperature. 


\section{Data availability}

The authors declare that the main data supporting the findings of this study are contained within the paper and its associated Supplementary Information. All other relevant data are available from the corresponding author upon reasonable request.

\section{Declarations}

\section{Acknowledgements}

$X$. Zhong is thankful to Jiuhua Chen for valuable discussions. This work was supported by the Major Program of the National Natural Science Foundation of China (Grant No. 52090024), National Natural Science Foundation of China (Grant No. 11534003, 11874175, 11874176, 12074138, and 11704151), Science and Technology Development Project of Jilin province under No. 20190103043JH, Fundamental Research Funds for the Central Universities (Jilin University, JLU), Program for JLU Science and Technology Innovative Research Team (JLUSTIRT), and The Strategic Priority Research Program of Chinese Academy of Sciences (Grant No. XDB33000000). This work used computing facilities at the High-Performance Computing Centre of Jilin Uni- versity. Calculations were also performed on computing facilities at HOKUSAI system in RIKEN (Japan).

\section{Author Contributions}

Y.M. and H.L. designed the research; X.Z., and Y.S. performed the calculations; X.Z., Y.S., T.I., M.X., H.L., C.C., and Y.M. analyzed and interpreted the data, and contributed to the writing of the paper.

$\$ X . Z$. and Y.S. equally contributed to this work.

†Electronic address: Ihy@calypso.cn

‡Electronic address: changfeng.chen@unlv.edu

§Electronic address: mym@jlu.edu.cn

\section{Competing interests}

The authors declare no competing interests. 


\section{Additional information}

Supplementary Information is available for this paper at $\mathrm{xxxxxx}$

Correspondence and requests for materials should be addressed to H.L., C.C., or Y.M.

Reprints and permission information is available online at $x x x x x$

\section{References}

1. Wigner, E. \& Huntington, H. B. On the possibility of a metallic modification of hydrogen. J. Chem. Phys. 3, 764-770 (1935).

2. Ashcroft, N. W. Metallic Hydrogen: A High-Temperature Superconductor? Phys. Rev. Lett. 21, 17481749 (1968).

3. Zhang, L. et al. Ab initio prediction of superconductivity in molecular metallic hydrogen under high pressure. Solid State Commun. 141, 610-614 (2007).

4. Cudazzo, P. et al. Ab initio description of high-temperature superconductivity in dense molecular hydrogen. Phys. Rev. Lett. 100, (2008).

5. McMahon, J. M. \& Ceperley, D. M. High-temperature superconductivity in atomic metallic hydrogen. Phys. Rev. B 84, (2011).

6. Dias, R. P. \& Silvera, I. F. Observation of the Wigner-Huntington transition to metallic hydrogen. https://www.science.org (2017).

7. Eremets, M. I., Drozdov, A. P., Kong, P. P. \& Wang, H. Semimetallic molecular hydrogen at pressure above 350 GPa. Nature Physics vol. 15 1246-1249 (2019).

8. Loubeyre, P., Occelli, F. \& Dumas, P. Synchrotron infrared spectroscopic evidence of the probable transition to metal hydrogen. Nature 577, (2020).

9. Satterthwaite, C. B. \& Toepke, I. L. Superconductivity of Hydrides and Deuterides of Thorium. Phys. Rev. Lett. 25, (1970).

10. Gilman, J. J. Lithium Dihydrogen Fluoride-An Approach to Metallic Hydrogen. Phys. Rev. Lett. 26, (1971).

11. Ashcroft, N. W. Hydrogen Dominant Metallic Alloys: High Temperature Superconductors? Phys. Rev. Lett. 92, (2004).

12. Zhang, L., Wang, Y., Lv, J. \& Ma, Y. Materials discovery at high pressures. Nat. Rev. Mater. 2, (2017).

13. Wang, H., Li, X., Gao, G., Li, Y. \& Ma, Y. Hydrogen-rich superconductors at high pressures. WIREs Comput. Mol. Sci. 8, (2018).

14. Flores-Livas, J. A. et al. A perspective on conventional high-temperature superconductors at high pressure: Methods and materials. Phys. Rep. 856, (2020). 
15. Drozdov, A. P., Eremets, M. I., Troyan, I. A., Ksenofontov, V. \& Shylin, S. I. Conventional

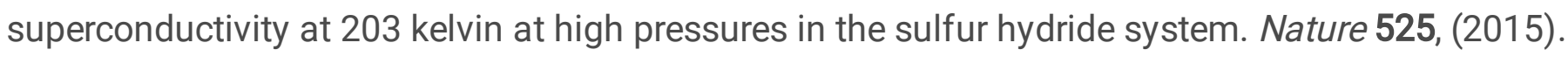

16. Li, Y., Hao, J., Liu, H., Li, Y. \& Ma, Y. The metallization and superconductivity of dense hydrogen sulfide. J. Chem. Phys. 140, (2014).

17. Duan, D. et al. Pressure-induced metallization of dense $\left(\mathrm{H}_{2} \mathrm{~S}\right)_{2} \mathrm{H}_{2}$ with high-Tc superconductivity. Sci. Rep. 4, (2015).

18. Li, Y. et al. Pressure-stabilized superconductive yttrium hydrides. Sci. Rep. 5, (2015).

19. Liu, H., Naumov, I. I., Hoffmann, R., Ashcroft, N. W. \& Hemley, R. J. Potential high- Tc superconducting lanthanum and yttrium hydrides at high pressure. Proc. Natl. Acad. Sci. 114, (2017).

20. Peng, F. et al. Hydrogen Clathrate Structures in Rare Earth Hydrides at High Pressures: Possible Route to Room-Temperature Superconductivity. Phys. Rev. Lett. 119, (2017).

21. Wang, H., Tse, J. S., Tanaka, K., litaka, T. \& Ma, Y. Superconductive sodalite-like clathrate calcium hydride at high pressures. Proc. Natl. Acad. Sci. 109, (2012).

22. Somayazulu, M. et al. Evidence for Superconductivity above $260 \mathrm{~K}$ in Lanthanum Superhydride at Megabar Pressures. Phys. Rev. Lett. 122, (2019).

23. Drozdov, A. P. et al. Superconductivity at $250 \mathrm{~K}$ in lanthanum hydride under high pressures. Nature 569, (2019).

24. P. P. Kong et al. Superconductivity up to $243 \mathrm{~K}$ in yttrium hydrides under high pressure. arXiv1909.10482 (2019).

25. Troyan, I. A. et al. Anomalous High-Temperature Superconductivity in $\mathrm{YH}_{6}$. Adv. Mater. 33, (2021).

26. Ma, L. et al. Experimental observation of superconductivity at $215 \mathrm{~K}$ in calcium superhydride under high pressures. arXiv:2103.16282 (2021).

27. Z.W.Li et al. Superconductivity above $200 \mathrm{~K}$ Observed in Superhydrides of Calcium. (2021).

28. Sun, Y., Lv, J., Xie, Y., Liu, H. \& Ma, Y. Route to a Superconducting Phase above Room Temperature in Electron-Doped Hydride Compounds under High Pressure. Phys. Rev. Lett. 123, (2019).

29. Liang, X. et al. Potential high-Tc superconductivity in $\mathrm{CaYH}_{12}$ under pressure. Phys. Rev. B 99, (2019).

30. Xie, H. et al. High-temperature superconductivity in ternary clathrate $\mathrm{YCaH}_{12}$ under high pressures. J. Phys. Condens. Matter 31, (2019).

31. Semenok, D. V. et al. Superconductivity at $161 \mathrm{~K}$ in thorium hydride $\mathrm{ThH} 10$ : Synthesis and properties. Mater. Today 33, (2020).

32. Kruglov, I. A. et al. Superconductivity of $\mathrm{LaH}_{10}$ and $\mathrm{LaH}_{16}$ polyhydrides. Phys. Rev. B 101, (2020).

33. Wang, X., Li, M., Zheng, F. \& Zhang, P. Crystal structure prediction of uranium hydrides at high pressure: A new hydrogen-rich phase. Phys. Lett. A 382, (2018).

34. Wang, Y., Lv, J., Zhu, L. \& Ma, Y. CALYPSO: A method for crystal structure prediction. Comput. Phys. Commun. 183, (2012). 
35. Wang, Y., Lv, J., Zhu, L. \& Ma, Y. Crystal structure prediction via particle-swarm optimization. Phys. Rev. B 82, (2010).

36. Supplemental Material provides details on computational methods used in this work to search for crystal structures of the $\mathrm{MH}_{18}$ compounds and to calculate electronic, phonon and superconducting properties of these extreme superhydrides, pressure driven changes of the band states and the effect of zero-point energy on structural stability, along with detailed structural data on two representative structures of $\mathrm{CeH}_{18}$ and $\mathrm{LaH}_{18}$ in the Fddd and $\mathrm{Fmmm}$ phases, respectively.

37. Eliashberg, G. M. Interactions between electrons and lattice vibrations in a superconductor. Sov. Phys. JETP 11, (1960).

38. A. B. Migdal. Interaction between electrons and lattice vibrations in a normal metal. Sov. Phys. JETP 34, undefined (1958).

39. Sanna, A. et al. Ab initio Eliashberg Theory: Making Genuine Predictions of Superconducting Features. J. Phys. Soc. Japan 87, (2018).

40. The elk code, http://elk.sourceforge.net/.

41. McMahon, J. M. \& Ceperley, D. M. Ground-State Structures of Atomic Metallic Hydrogen. Phys. Rev. Lett. 106, (2011).

42. Snider, E. et al. Synthesis of Yttrium Superhydride Superconductor with a Transition Temperature up to $262 \mathrm{~K}$ by Catalytic Hydrogenation at High Pressures. Phys. Rev. Lett. 126, (2021).

43. Kennedy, J. \& Eberhart, R. Particle swarm optimization. Proc. ICNN'95 - Int. Conf. Neural Networks doi:10.1109/ICNN.1995.488968.

44. Eberhart, R. \& Kennedy, J. New optimizer using particle swarm theory. in Proceedings of the International Symposium on Micro Machine and Human Science 39-43 (IEEE, 1995). doi:10.1109/mhs.1995.494215.

45. Gao, B. et al. Interface structure prediction via CALYPSO method. Sci. Bull. 64, (2019).

46. Kresse, G. \& Furthmüller, J. Efficient iterative schemes for ab initio total-energy calculations using a plane-wave basis set. Phys. Rev. B 54, (1996).

47. Perdew, J. P. \& Wang, Y. Accurate and simple analytic representation of the electron-gas correlation energy. Phys. Rev. B 45, (1992).

48. Perdew, J. P., Burke, K. \& Ernzerhof, M. Generalized Gradient Approximation Made Simple. Phys. Rev. Lett. 77, (1996).

49. Togo, A., Oba, F. \& Tanaka, I. First-principles calculations of the ferroelastic transition between rutiletype and $\mathrm{CaCl}_{2}$-type $\mathrm{SiO}_{2}$ at high pressures. Phys. Rev. B. 78, (2008).

50. Giannozzi, P. et al. QUANTUM ESPRESSO: a modular and open-source software project for quantum simulations of materials. J. Phys. Condens. Matter 21, (2009).

51. Allen, P. B. \& Mitrović, B. Theory of Superconducting TC. in (1983). doi:10.1016/S00811947(08)60665-7.

52. Pickard, C. J. \& Needs, R. J. Structure of phase III of solid hydrogen. Nat. Phys. 3, (2007). 


\section{Table}

Calculated pressure (P, GPa) variation of $\lambda, \omega_{\text {log }}(\mathrm{K}), N\left(E_{\mathrm{f}}\right)$ (states/Ry/f.u./spin) and $T_{c}(\mathrm{~K})$ for $\mu^{\star}=0.10(0.13)$ for selected $\mathrm{MH}_{18}$ compounds in Fmmm or Fddd phase [(M) indicates metastable phase] and hydrogen in $I_{1} /$ amd phase. The values of $T_{c}$ for $F d d d-\mathrm{CeH}_{18}$ and $F m m m-\mathrm{ThH}_{18}$ with the inclusion of SOC are also listed. 


\begin{tabular}{|c|c|c|c|c|c|c|}
\hline Compound & $\mathrm{P}$ & $\lambda$ & $\omega_{\log }$ & $N\left(E_{f}\right)$ & $T_{\mathrm{c}}$ & $T_{\mathrm{c}}(\mathrm{SOC})$ \\
\hline $\mathrm{H}-14_{1} / \operatorname{amd}(\mathrm{M})$ & 300 & 2.34 & 1577 & 0.23 & $339(320)$ & - \\
\hline $\mathrm{H}-14_{1} / \operatorname{amd}(\mathrm{M})$ & 400 & 2.39 & 1719 & 0.25 & $383(362)$ & - \\
\hline $\mathrm{H}-14_{1} /$ amd & 500 & 2.24 & 1806 & 0.23 & $380(356)$ & - \\
\hline $\mathrm{H}-14_{1} / \mathrm{amd}$ & 600 & 2.10 & 1953 & 0.23 & $388(362)$ & - \\
\hline $\mathrm{H}-14_{1} / \mathrm{amd}$ & 700 & 2.08 & 2021 & 0.25 & $401(375)$ & - \\
\hline $\mathrm{YH}_{18}-$ Fddd & 500 & 1.50 & 1082 & 4.21 & 183(165) & - \\
\hline $\mathrm{YH}_{18}-$ Fddd & 600 & 1.28 & 1467 & 3.96 & 176(158) & - \\
\hline $\mathrm{YH}_{18^{-}}$Fddd & 700 & 1.10 & 1704 & 3.74 & $162(140)$ & - \\
\hline $\mathrm{LaH}_{18}-\mathrm{Fddd}$ & 600 & 0.74 & 1638 & 3.05 & 70(55) & - \\
\hline $\mathrm{LaH}_{18}{ }^{-F d d d}$ & 700 & 0.78 & 1642 & 3.21 & 79(64) & - \\
\hline $\mathrm{LaH}_{18}-\mathrm{Fmmm}$ & 300 & 2.46 & 1156 & 4.66 & $271(255)$ & - \\
\hline $\mathrm{LaH}_{18}-\mathrm{Fmmm}$ & 350 & 1.68 & 1491 & 4.40 & $239(222)$ & - \\
\hline $\mathrm{LaH}_{18}-\mathrm{Fmmm}$ & 400 & 1.28 & 1649 & 4.13 & 192(174) & - \\
\hline $\mathrm{LaH}_{18}-F m m m$ & 500 & 0.82 & 1663 & 3.23 & $93(76)$ & - \\
\hline $\mathrm{CeH}_{18}-\mathrm{Fddd}(\mathrm{M})$ & 300 & 2.03 & 985 & 5.04 & $223(207)$ & $224(208)$ \\
\hline $\mathrm{CeH}_{18}-\mathrm{Fddd}$ & 350 & 2.80 & 919 & 6.04 & $329(309)$ & $330(310)$ \\
\hline $\mathrm{CeH}_{18}-\mathrm{Fddd}$ & 400 & 2.32 & 1294 & 6.19 & $312(292)$ & $310(290)$ \\
\hline $\mathrm{CeH}_{18}-\mathrm{Fddd}$ & 500 & 1.85 & 920 & 5.09 & $240(212)$ & - \\
\hline $\mathrm{CeH}_{18}-\mathrm{Fddd}$ & 600 & 1.64 & 844 & 4.88 & 216(194) & - \\
\hline $\mathrm{CeH}_{18}-F m m m(\mathrm{M})$ & 400 & 2.21 & 1260 & 4.17 & $285(265)$ & - \\
\hline $\mathrm{CeH}_{18}-F m m m$ & 600 & 1.76 & 1388 & 4.20 & $285(262)$ & - \\
\hline $\mathrm{AcH}_{18}-F d d d$ & 400 & 0.92 & 1411 & 3.45 & $99(83)$ & - \\
\hline $\mathrm{AcH}_{18}-\mathrm{Fddd}$ & 500 & 0.80 & 1480 & 3.31 & $76(62)$ & - \\
\hline $\mathrm{AcH}_{18}-\mathrm{Fddd}$ & 600 & 0.65 & 1718 & 3.29 & $53(40)$ & - \\
\hline
\end{tabular}




\begin{tabular}{|lcccccl|}
$\mathrm{AcH}_{18}-\mathrm{Fddd}$ & 700 & 0.62 & 1844 & 3.43 & $50(37)$ & - \\
\hline $\mathrm{AcH}_{18}{ }^{-}-\mathrm{Fmmm}$ & 300 & 1.68 & 1217 & 4.39 & $206(190)$ & - \\
\hline $\mathrm{AcH}_{18}-\mathrm{Fmmm}$ & 400 & 1.36 & 904 & 3.73 & $134(119)$ & - \\
\hline $\mathrm{ThH}_{18}-$ Fddd & 300 & 1.15 & 1227 & 4.01 & $131(116)$ & - \\
\hline $\mathrm{ThH}_{18}-\mathrm{Fmmm}$ & 400 & 3.39 & 568 & 7.09 & $296(277)$ & $296(277)$ \\
\hline $\mathrm{ThH}_{18}-\mathrm{Fmmm}(\mathrm{M})$ & 500 & 1.92 & 1573 & 7.55 & $306(284)$ & $306(285)$ \\
\hline $\mathrm{ThH}_{18}{ }^{-}-\mathrm{Fmmm}(\mathrm{M})$ & 600 & 2.13 & 1331 & 7.92 & $321(299)$ & $321(299)$ \\
\hline
\end{tabular}

\section{Figures}
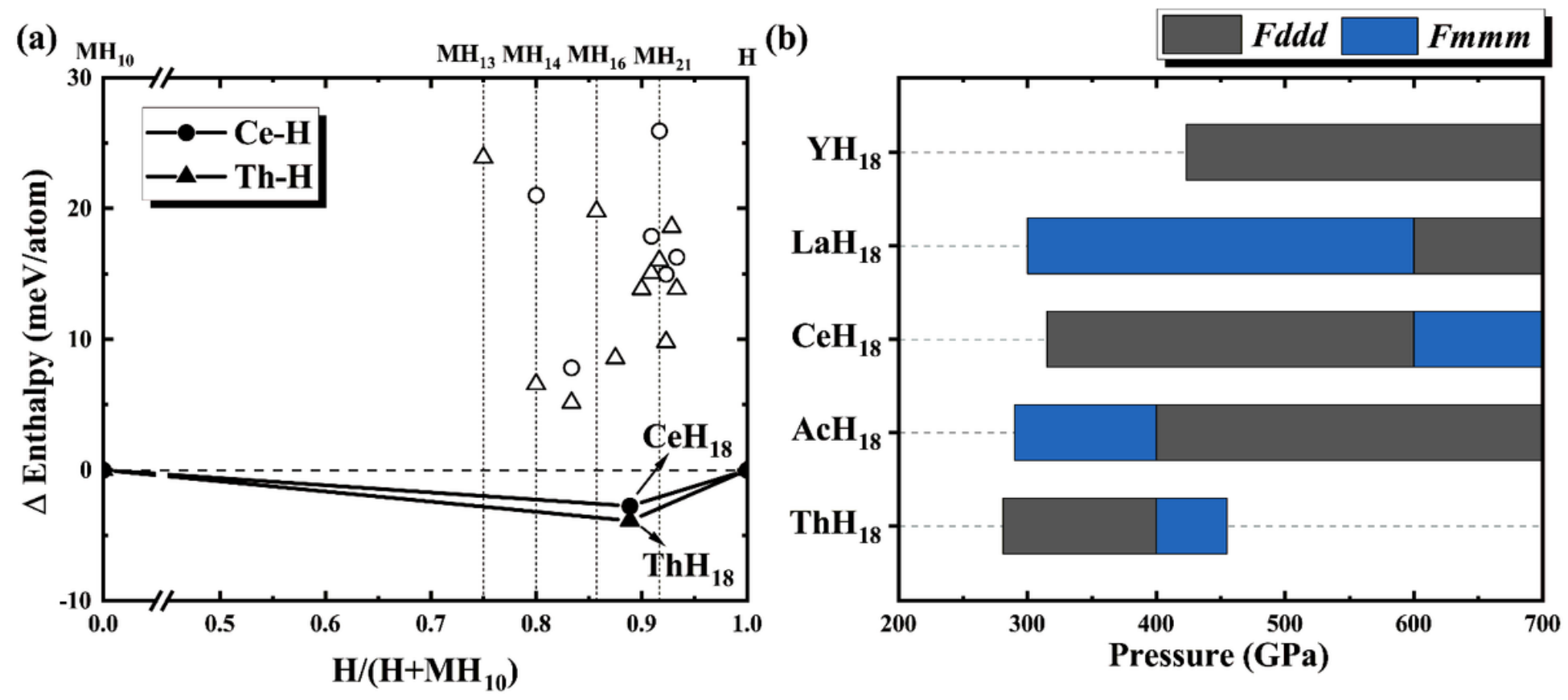

Figure 1

(a) Calculated convex-hull of newly identified extreme superhydrides $\mathrm{CeH} 18$ and ThH18, both in the Fddd phase, with respect to decomposition into Ce/ThH1020, 31 and H241, 52 at $400 \mathrm{GPa}$. Data points located on the convex hull (solid lines) represent stable species against possible decomposition. The open symbols represent some metastable/unstable compositions identified by the search process. (b) Stable pressure ranges of the predicted series of extreme superhydrides $\mathrm{MH} 18$ with respect to decomposition into known $\mathrm{MHx}(\mathrm{x}=2,4,6,9,10,12,16$, and 17$)$ and $\mathrm{Cmca}-\mathrm{H} 2$, and these results are obtained via calculations with the inclusion of the zero-point energy (ZPE). 

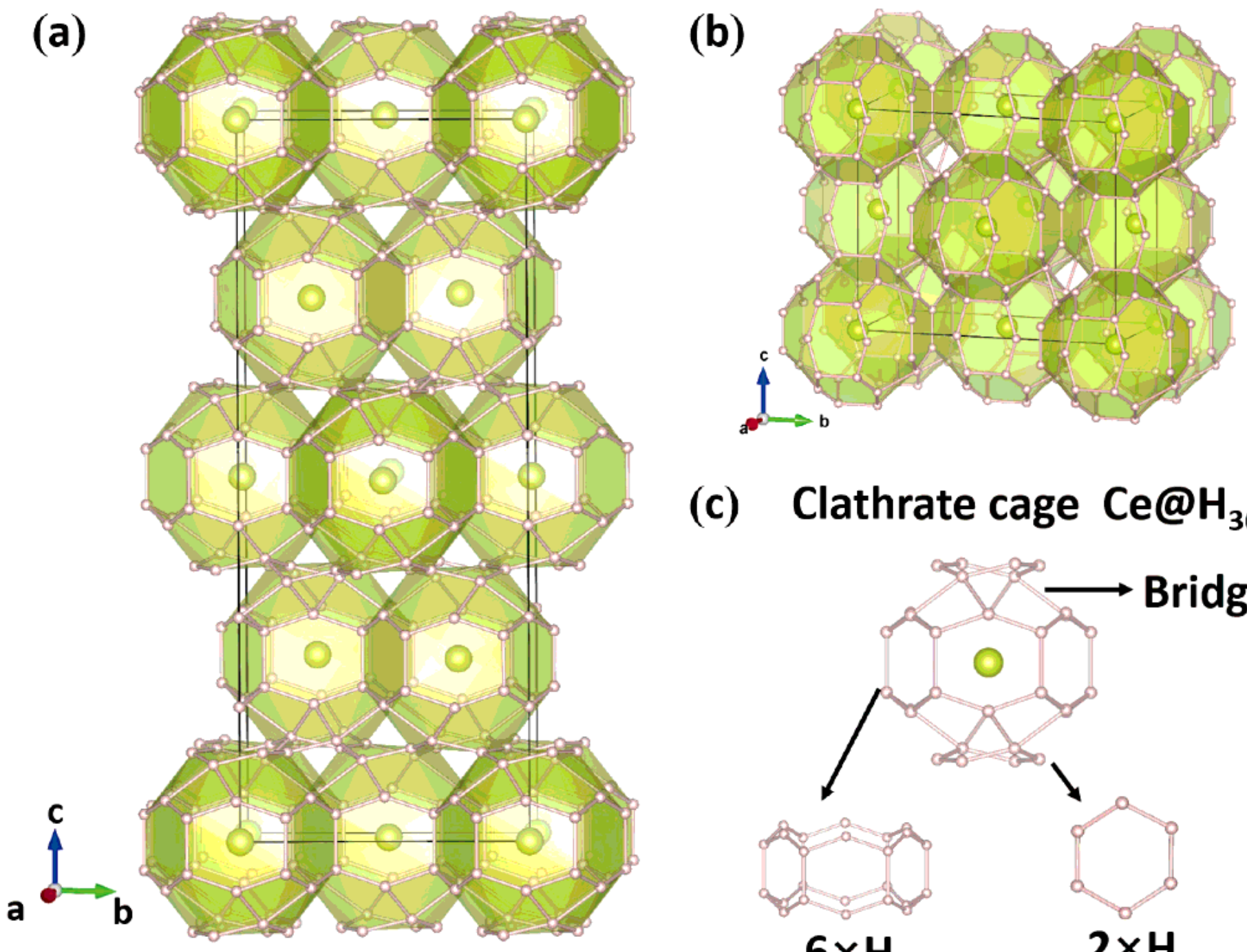

\section{(c) Clathrate cage $\mathrm{Ce}_{36}$}

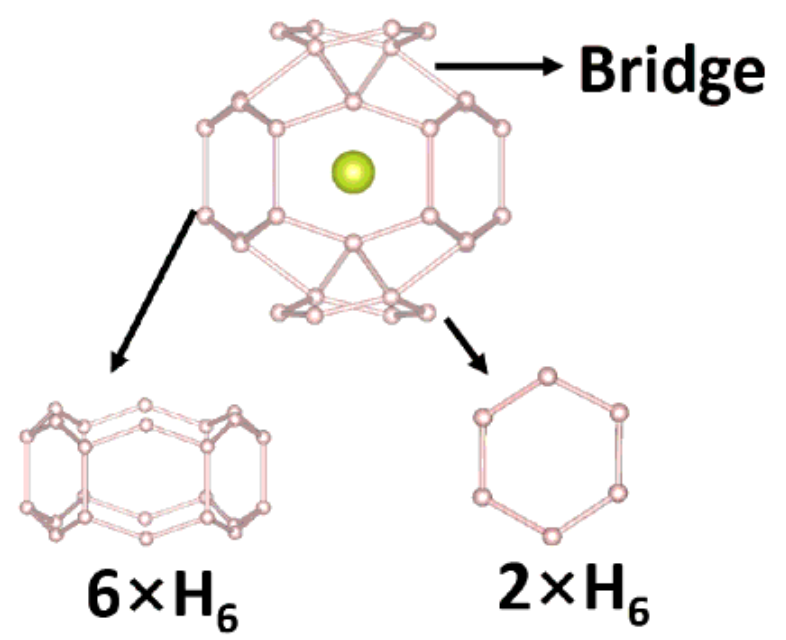

Figure 2

The crystal structure of MH18 in (a) Fddd and (b) Fmmm phase. (c) The building units of the Ce@H36 hydrogen clathrate cage, including a $6 \mathrm{H} 6$ ribbon-ring and two $\mathrm{H} 6$ hexagons, which are connected by pertinent bridge bonding networks. The large and small spheres represent the metal and hydrogen atoms, respectively. 
(a)
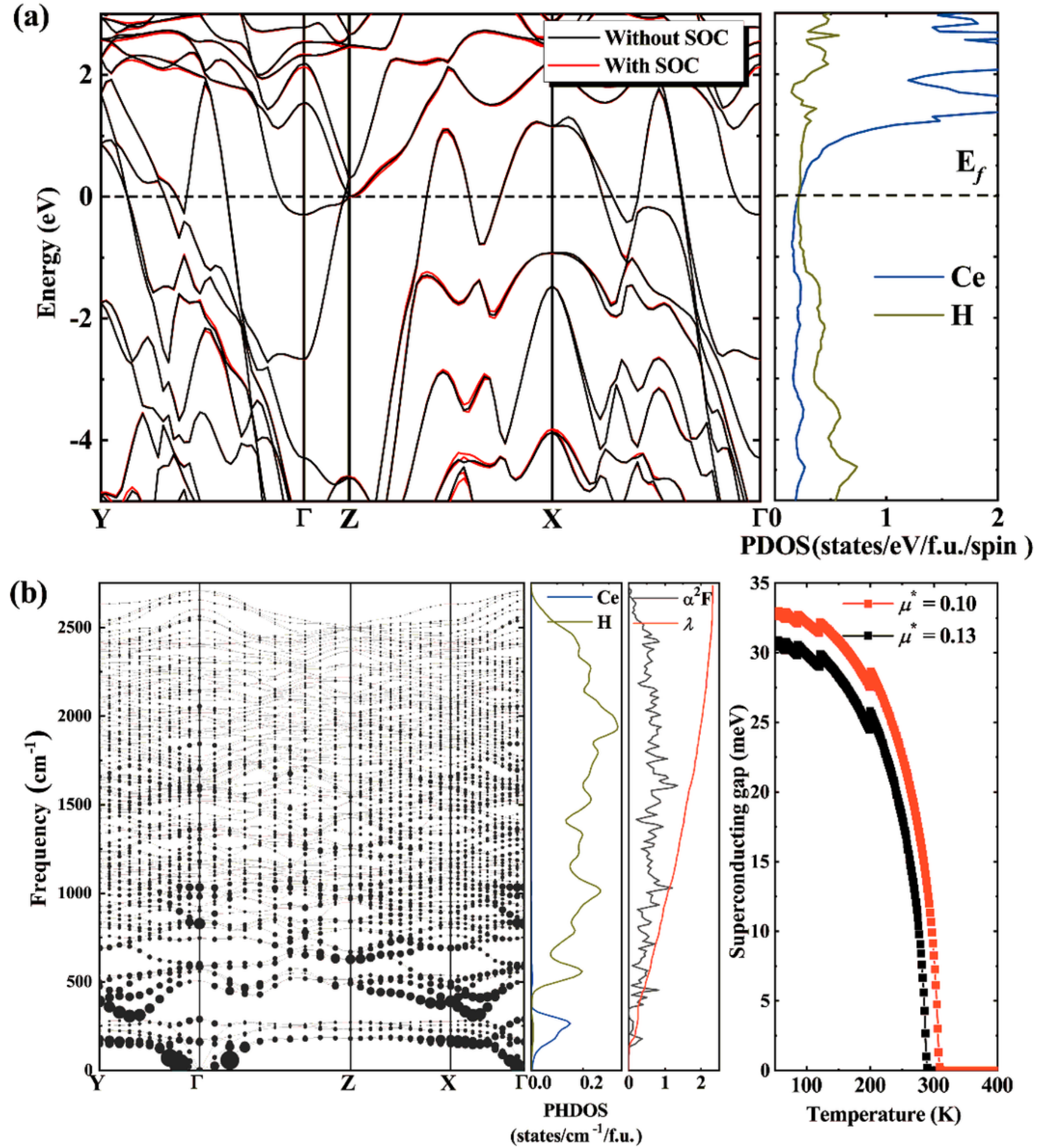

Figure 3

(a) Electronic band structure (left) and projected density of states (right) of CeH18 at $400 \mathrm{GPa}$. The band structures without and with the inclusion of SOC are plotted with black and red lines, respectively. (b) Phonon dispersion curves with the strength of q resolved $\lambda q$ indicated by circle size (left), phonon density of states (PHDOS), Eliashberg spectral function a2F $(\omega)$ and EPC parameter $\lambda(\omega)$ (middle), and superconducting energy gap (right) of $\mathrm{CeH} 18$ at $400 \mathrm{GPa}$. 


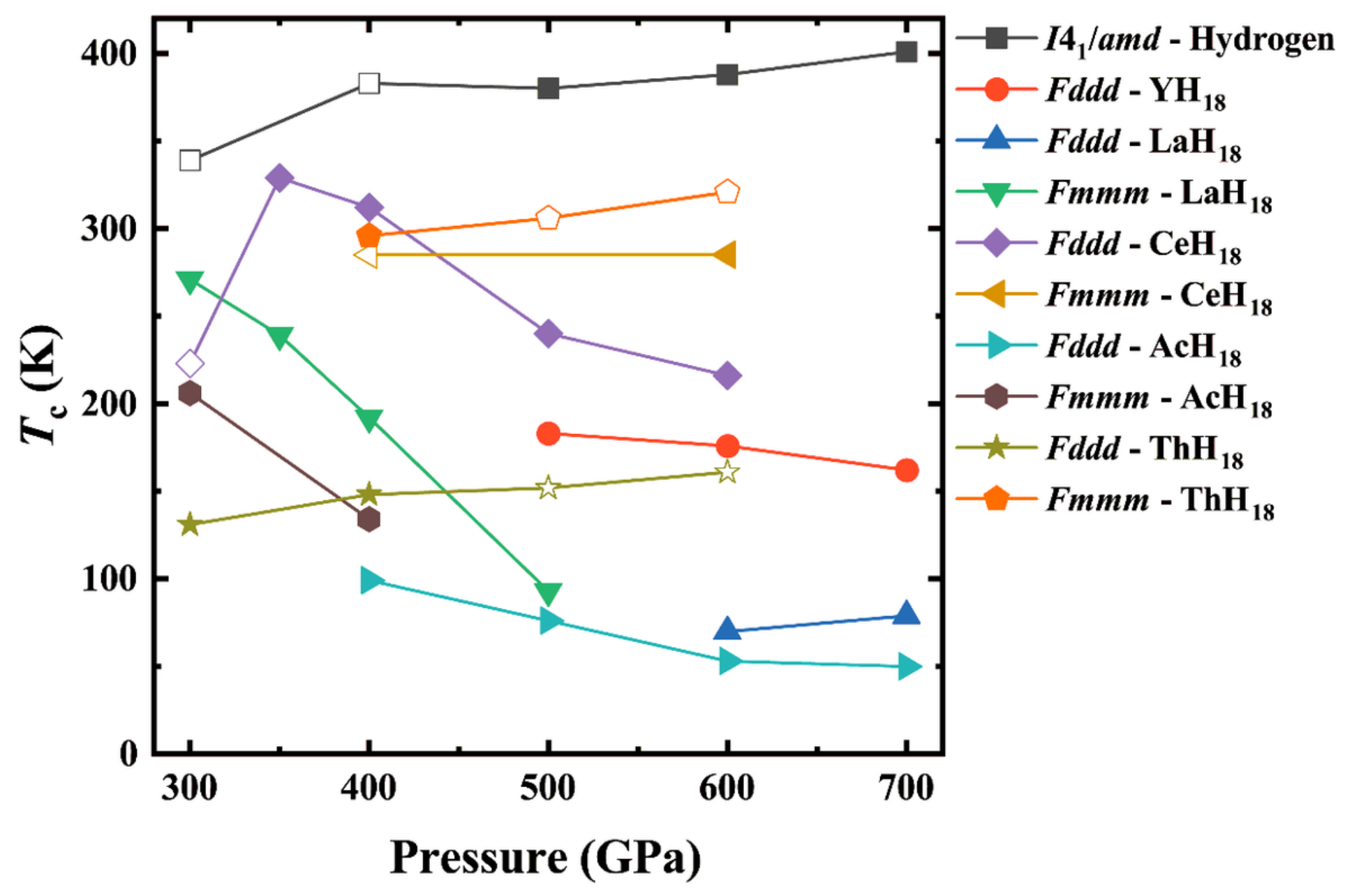

Figure 4

Calculated Tc $(\mu \star=0.10)$ for selected extreme superhydrides MH18 compared with the results for the 141/amd phase of solid hydrogen at high pressures. Solid (open) symbols represent the Tc data for stable (metastable) structural phases of the indicated MH18 compounds and solid hydrogen.

\section{Supplementary Files}

This is a list of supplementary files associated with this preprint. Click to download.

- SMMH18NCzxhy.docx 